\title{
There but for the grace of God ...
}

\author{
Fiona Godlee editor in chief
}

The BMJ

Who of us has not made mistakes that have harmed patients? Some years ago I and other senior doctors were asked to write about a serious error we had made (doi:10.1136/bmj.331.7517. 595-b; http://bit.ly/2DOHE1K).

I'm sure each of us had many to choose from. I wrote about a patient in whom I correctly diagnosed necrotising fasciitis but then sent to a general ward rather than intensive care. The patient survived but only after arresting and losing fingers and toes from disseminated intravascular coagulation. The then president of the UK General Medical Council, Graeme Catto, told how he still felt guilty because, as a newly appointed consultant, he delayed starting antibiotics in a young man with meningococcal septicaemia. The patient died.

Reflecting on the criminal conviction and now striking off of Hadiza Bawa-Garba for gross negligence manslaughter (doi:10. 1136/bmj.k456; doi:10.1136/bmj.k417), the consultant cardiologist Peter Wilmshurst has gone one big step further. Renowned for referring others to the GMC, he has now referred himself and encourages others to do the same (bmj.com/content/ 360/bmj.k417/rr-5; doi:10.1136/bmj.k481). "During four decades of practice, I have made clinical errors including delayed diagnosis and errors in treatment," he writes. "In some cases my errors are likely to have contributed to poor outcomes and some patient deaths."

Self referral may seem extreme, but like other protests against the GMC's decision it shows the depth of concern among doctors. Those working on the front line are justifiably worried that honest errors can now end their careers. As Margaret McCartney writes, a culture of fear looms (doi:10.1136/bmj. $\mathrm{k} 443$ ). Doctors seem now to be expected to have limitless capabilities, and the crucial role of human factors and systemic failures is pushed aside.

Understaffing and inadequate support make human error even more likely. David Oliver says the GMC has no clear guidance on whether doctors should refuse to work in unsafe conditions (doi:10.1136/bmj.k448). Doctors are advised to use their own judgment if they think patients, or their own wellbeing, are at risk. However broken the systems, doctors still risk being held personally culpable for failings. This is a far cry, Oliver says, from the airline industry's rigorous safeguards. However broken the systems, doctors still risk being held personally culpable for failings.

Heigi Johannson and William Rook write that medical law needs the same transformation as medical practice has undergone regarding liability and error (doi:10.1136/bmj.k447). And in an editorial we call for action to avoid individual blame and criminalisation of health professionals (doi:10.1136/bmj.k479): "It is tragic that a child has died. But no one is served when one doctor is blamed for the failings of an overstretched and understaffed system. We must channel the sadness at Jack Adcock's death, and the anger at Bawa-Garba's fate, into positive change for safer patient care." 